\title{
Electrical transport in carbon nanotubes: Role of disorder and helical symmetries
}

\author{
François Triozon, ${ }^{1, *}$ Stephan Roche, ${ }^{1, \dagger}$ Angel Rubio, ${ }^{2}$ and Didier Mayou ${ }^{3}$ \\ ${ }^{1}$ Commissariat à l'Energie Atomique, DSM/DRFMC/SPSMS and DRT/LETI/DIHS/LMNO, 17 rue des Martyrs, \\ 38054 Grenoble Cedex 9, France \\ ${ }^{2}$ Departamento de Física de Materiales, Universidad del Pais Vasco, Centro Mixto CSIC-UPV/EHU and Donostia International Physics \\ Center (DIPC), Apartado 1072, 20018 San Sebastián, Spain \\ ${ }^{3}$ LEPES-CNRS, 25 rue des Martyrs, Boîte Postale 166, 38042 Grenoble Cedex 9, France
}

(Received 9 January 2004; published 24 March 2004)

\begin{abstract}
For carbon nanotubes, we determine the role of disorder and helicity in the transport length scales and intrinsic conductance. Our results highlight different physical phenomena originating from defect scattering and multishell conduction. Those effects are sensitive to the position of the Fermi level, and allow for a consistent interpretation of recent transport experiments in doped nanotubes.
\end{abstract}

DOI: 10.1103/PhysRevB.69.121410

PACS number(s): 73.63.Fg, 71.15.-m

Helical symmetries in carbon nanotubes are at the spectacular origin of a metallic versus semiconducting electronic status. ${ }^{1}$ Metallic single-wall nanotubes (SWNT's), with their Fermi level at the charge neutrality point (CNP), have been further demonstrated to behave as exceptionally long ballistic conductors, due to a vanishing contribution of elastic backscattering (pseudospin symmetry conservation), 2,3 and upscaling of the mean free path with nanotube diameter was derived in the framework of the Fermi golden rule. ${ }^{4}$

Multiwall nanotubes (MWNT's) are made from a few to several tens of weakly coupled concentric shells, separated by few angstroms and with random helicities. Although ballistic conduction has been reported in several experiments in SWNT's and MWNT's, ${ }^{3,5,6}$ the bias dependence of the conductance in MWNT's highlights the possible role of disorder and/or intershell conduction. ${ }^{7-11}$ At large bias, the contribution of several subbands is expected, with an enhanced backscattering for the so-called massive subbands, as compared to the two massless subbands crossing at the $\mathrm{CNP}^{3}$ On the other hand, it has been argued theoretically that incommensurability between coupled shells can drive conduction to either diffusive, ballistic, or intermediate transport regime. ${ }^{12-15}$ Such scenario has been debated in subsequent works, restricted, however, to qualitative arguments or numerical computation on small systems. ${ }^{14,15}$ As it is now experimentally possible to have a simultaneous access to the helicity and transport phenomena in MWNT's, ${ }^{16}$ it is important to better clarify the fundamental length scales and transport regimes. Besides, the issue of electronic mobilities in carbon nanotube based field effect transistors is also of great concern. $^{17}$

In this work, the relative effect of elastic scattering on massless and massive subbands is quantified, in disordered nanotubes as long as $10 \mu \mathrm{m}$. This is performed by computing the energy dependence of diffusion coefficients and length scaling of the Kubo conductance, using a real-space numerical approach. Two sources of elastic scattering are considered separately. First, the effect of static (Andersonlike) disorder is studied in SWNT's and commensurate MWNT's. Second, multishell conduction is shown to be sensitive to incommensurability, which introduces scattering centers in otherwise structurally perfect MWNT's.
Transport is controlled by low-energy excitations close to the Fermi level $E_{F}$, which turn out to be well described by a simple tight-binding Hamiltonian treating only the coupling between $\pi$ electrons: ${ }^{18}$

$$
\hat{H}=\gamma_{0} \sum_{\langle i, j\rangle}\left|\pi_{i}\right\rangle\left\langle\pi_{j}\left|-\beta \sum_{\langle\langle i, j\rangle\rangle} \cos \left(\theta_{i j}\right) \exp \left(\frac{a-d_{i j}}{\delta}\right)\right| \pi_{i}\right\rangle\left\langle\pi_{j}\right| .
$$

The first term represents the intrashell coupling, with nearest-neighbor hopping $\gamma_{0} \simeq 3 \mathrm{eV}$. ${ }^{1}$ The second term represents the weak intershell coupling, which depends on the distance $d_{i j}$ and angle $\theta_{i j}$ between the $\pi_{i}$ and $\pi_{j}$ orbitals. The intershell interaction parameters are taken from firstprinciples results: $\quad \beta=\gamma_{0} / 8, \quad a=0.334 \mathrm{~nm}, \quad$ and $\delta$ $=0.045 \mathrm{~nm}^{18}$ Disorder is included through the general Anderson random fluctuations of the on-site energies, allowing for a direct comparison with the fermi golden rule approximation in its range of applicability. ${ }^{4}$ This model is a first approximation for studying the effect of disorder over mesoscopic length scales, although the atomistic structure of defects is not considered. Other studies have considered topological or chemical defects that introduce a different (offdiagonal) type of disorder. ${ }^{19}$ Note that intershell coupling in MWNT's is a form of off-diagonal disorder too. The combined description of charge transfer and defects, including chemical impurities, would require a first-principle description which is beyond the scope of the present work. ${ }^{20}$

The key quantity of our study is the time-dependent diffusion coefficient $\mathcal{D}(E, t)$, from which the Kubo conductance scaling follows. ${ }^{21}$ One defines

$$
\mathcal{D}(E, t)=\frac{\mathcal{X}^{2}(E, t)}{t}=\frac{1}{t} \frac{\operatorname{Tr}\left\{[\hat{X}(t)-\hat{X}(0)]^{2} \delta(E-\hat{H})\right\}}{\operatorname{Tr}[\delta(E-\hat{H})]}
$$

with $\mathcal{X}^{2}(E, t)$ the mean-square displacement of wave packets at energy $E$, after propagation during a time $t$. It is defined by the right-hand fraction, where $\hat{X}(t)$ is the position operator along the tube axis, in the Heisenberg representation, while $\delta(E-\hat{H})$ is the projection operator at energy $E . \mathcal{D}(E, t)$ thus represents wave-packet dynamics due to intrinsic properties 
of the nanotube. It can be further related to the conductance between two reflectionless contacts at a distance $L:^{21}$

$$
\mathcal{G}(E, L)=\frac{2 e^{2}}{L} \operatorname{Tr}[\delta(E-\hat{H})] \mathcal{D}\left(E, \tau_{L}\right)
$$

where $E$ is the Fermi energy, while $\tau_{L}$ is the propagation time over the distance $L$, defined by a condition on the meansquare displacement: $\left[\mathcal{X}^{2}\left(E, \tau_{L}\right)\right]^{1 / 2}=L$. For a ballistic electronic propagation, $\mathcal{X}^{2}(E, t) \propto t^{2}$, the conductance is length independent and equal to $N(E) \mathcal{G}_{0}$, where $N(E)$ is the number of conducting channels at energy $E$ and $\mathcal{G}_{0}=2 e^{2} / h$ is the quantum of conductance. In presence of elastic scattering, propagation becomes sub-ballistic and the conductance is always smaller than the ideal limit $N(E) \mathcal{G}_{0}$. Note that the relevant energy $E$ is not necessarily located at the CNP since chemical impurities or gate voltage might shift the Fermi level position. ${ }^{22}$ Besides, when the bias voltage is large, electronic excitations far from the Fermi level also contribute to transport. Thus, studying the energy dependence of the diffusion coefficient $\mathcal{D}(E, t)$ is of clear significance for a full understanding of experiments.

In practice, $\mathcal{D}(E, t)$ has to be calculated in a system which is longer than $L$. To perform energy-dependent calculations of diffusion coefficients for SWNT's and MWNT's as long as $10 \mu \mathrm{m}$, our previous real-space numerical method ${ }^{12}$ has been improved to cope with this large scale simulation (in size and time propagation). The idea is to rewrite the numerator of Eq. (2) as $\operatorname{Tr}\left\{[\hat{X}, \hat{U}(t)]^{\dagger} \delta(E-\hat{H})[\hat{X}, \hat{U}(t)]\right\}$, where $[\hat{X}, \hat{U}(t)]$ is the commutator of $\hat{X}$ with the evolution operator $\hat{U}(t)=\exp (-i \hat{H} t / \hbar)$. The trace is efficiently computed through an average over random-phase states, namely, $N \times\left\langle\psi\left|[\hat{X}, \hat{U}(t)]^{\dagger} \delta(E-\hat{H})[\hat{X}, \hat{U}(t)]\right| \psi\right\rangle$, where $N$ is the number of orbitals, and the $|\psi\rangle$ are normalized states extended to the whole nanotube, with constant modulus and random phase on each orbital. $|\psi\rangle$ contains all energy components, and for the large systems considered here, one or few different states $|\psi\rangle$ will be sufficient to obtain a good estimation of the trace. ${ }^{21,23}$ Given a random-phase state $|\psi\rangle$, the evolution of $[\hat{X}, \hat{U}(t)]|\psi\rangle$ is computed by using the Chebyshev development of $\hat{U}(t)$ (Ref. 24) and by imposing periodic boundary conditions, as in Ref. 23. Finally, at selected time steps, the "local density of states" (LDOS) of the vector $[\hat{X}, \hat{U}(t)]|\psi\rangle$ is obtained by a continued fraction method. This approach is very fast and allows to obtain the LDOS over the whole spectrum with an energy resolution of $0.5 \%$ of the spectrum bandwidth. If necessary, the entire procedure is repeated for different states $|\psi\rangle$, and the numerator of Eq. (2) is obtained by averaging all the LDOS. The continued fraction method is also used to calculate the denominator of Eq. (2), by averaging the LDOS of random-phase states. Once all these data are accumulated for a sufficient number of time steps $t$, the evolution of the diffusion coefficient $\mathcal{D}(E, t)$ can be deduced, as well as the transport properties, for any value of the Fermi energy $E$.

First, several SWNT's with Anderson disorder are considered. The on-site energies fluctuate between $-W / 2$ and $W / 2$ (in units of the hopping energy $\gamma_{0}$ ), with a uniform statistical

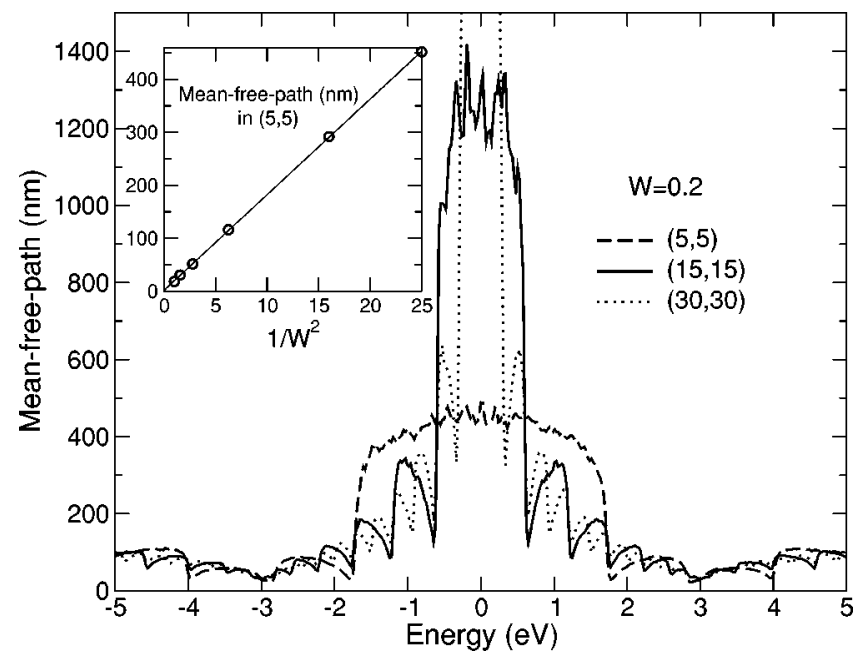

FIG. 1. Energy dependence of the mean free path in several armchair nanotubes with disorder $W=0.2$. The inset shows the scaling of $l_{e}$ with $W$ for the $(5,5)$ tube.

distribution. The diffusion coefficient evolves ballistically at short times: $\mathcal{D}(E, t) \simeq v^{2}(E) t$, where $v(E)$ is the average wave-packet velocity at energy $E$. It further saturates due to elastic scattering, and decreases owing to quantum interference effects. The elastic mean-free-path is commonly defined as

$$
l_{e}(E)=\frac{\mathcal{D}_{\text {max }}(E)}{v(E)},
$$

where $\mathcal{D}_{\text {max }}(E)$ is the maximum of the diffusion coefficient. In Fig. 1, the energy dependence of the mean free path is shown for several armchair nanotubes, with the same disorder parameter $W=0.2$. For the $(5,5)$ tube at $E=0$, one gets $l_{e} \simeq 450 \mathrm{~nm}$, which is slightly underestimated in regard to the Fermi golden rule (FGR) which gives $550 \mathrm{~nm} .^{4}$ At this energy, the scaling of $l_{e}$ with $W$ and with the tube radius $R$ predicted by the FGR (Ref. 4) is correctly reproduced: $l_{e}$ $\sim\left(\gamma_{0} / W\right)^{2} R$. But the most important feature is the variation of $l_{e}$ with energy. The massive subbands are found to be more sensitive to disorder than the massless ones, in agreement with experiments on defected tubes. ${ }^{3}$ To investigate the effect of disorder on a single massive subband, the mean free path in the semiconducting tube $(8,0)$ is reported in Fig. 2, together with the conductance (inset), calculated using Eq. (3), for an interelectrode distance $L=200 \mathrm{~nm}$. When compared to the perfect tube, the conductance is much reduced by disorder, in particular close to the Van Hove singularity. The mean free path and conductance of $(5,5)$ are also shown for the same parameters $W$ and $L$. In this case, the quantized conductance is hardly affected by disorder. We note that inelastic scattering such as electron-phonon coupling has been shown to be inefficient at low temperature, ${ }^{25}$ whereas electron-electron scattering might play an important role in presence of metallic contacts with weak transparency (which is not the case considered here). ${ }^{26}$

In MWNT's, electronic transport is not only affected by scattering on the outer tube, but also, possibly, by the cou- 


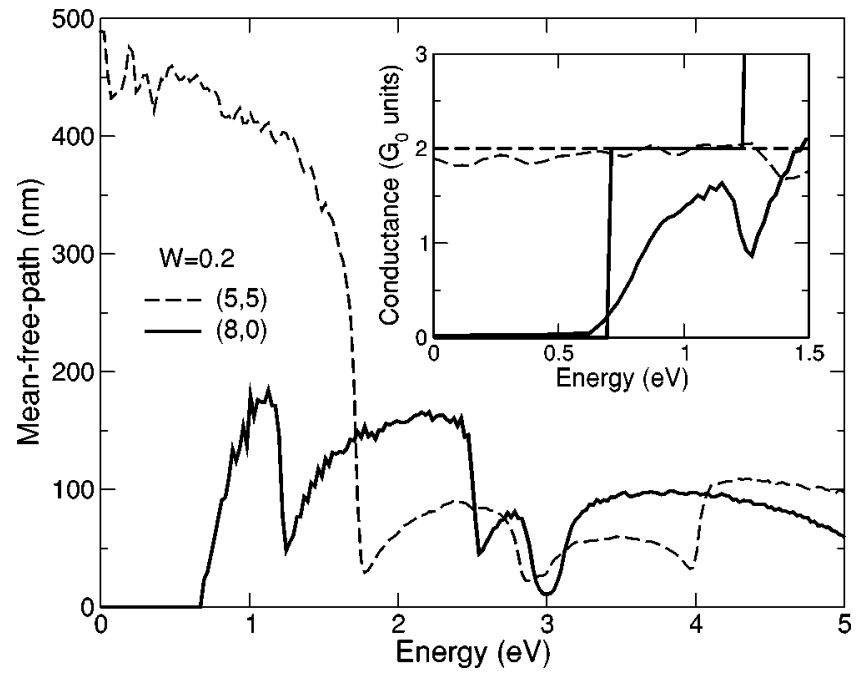

FIG. 2. Mean free path in the metallic $(5,5)$ and semiconducting $(8,0)$ nanotubes, with $W=0.2$. The inset shows the corresponding conductances for a distance between electrodes $L=200 \mathrm{~nm}$, as compared to the quantized conductance of perfect tubes. In the metallic band of $(5,5)$, the conductance is weakly reduced by disorder. In the first semiconducting band of $(8,0)$, disorder reduces the conductance well below the value $2 \mathcal{G}_{0}$ of the defect-free nanotube, particularly for energies close to the Van Hove singularity.

pling with one or several inner shells. To illustrate this effect, we consider the coupling of a disordered $(10,10)$ tube to a perfect $(5,5)$ tube. This corresponds to the experimental situation where impurities are adsorbed on the outer tube, while the inner metallic shells are preserved from contamination. From the behavior of diffusion coefficients represented in Fig. 3, the mean free path is remarkably shown to be increased (by more than a factor of 2) in presence of intershell coupling. In this case, intershell coupling with the perfect

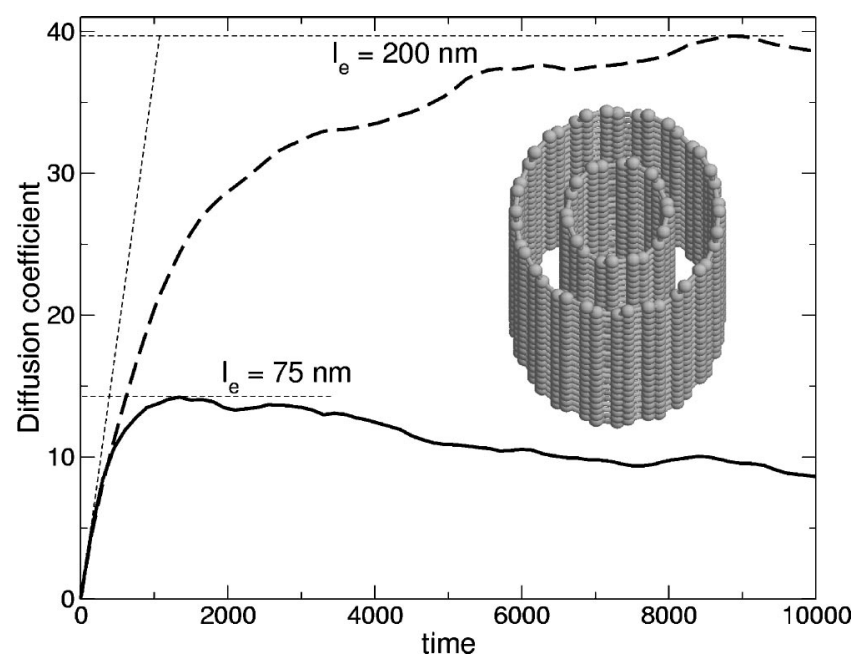

FIG. 3. Time-dependent diffusion coefficient at $E=0$ in a $(10,10)$ tube with $W=0.5$, isolated (bold line), or coupled to a perfect $(5,5)$ via intershell interaction (dashed line). The mean free path $l_{e}$ is calculated from the maximum value of $D(E=0, t)$ and from its slope at $t=0$, using Eq. (4). Time is in units of $\hbar / \gamma_{0}$ and the diffusion coefficient in $\mathrm{nm}^{2} \times \gamma_{0} / \hbar$.



FIG. 4. Left: time-dependent diffusion coefficient in a commensurate (bold line) and an incommensurate (dashed line) MWNT. Right: length-dependent conductance for the incommensurate tube (inset). Energy $E$ is in units of $\gamma_{0}$, time in units of $\hbar / \gamma_{0}$, and the diffusion coefficient in $\mathrm{nm}^{2} \times \gamma_{0} / \hbar$.

inner tube makes the system globally less disordered. This explains why the double wall maintains ballistic conduction over a longer scale, when compared to the individual disordered $(10,10)$.

Another controversial phenomenon is the role of intershell coupling in disorder-free incommensurate MWNT's. Incommensurability between two adjacent shells takes place whenever the ratio of their respective lengths along the tube axis is an irrational number. ${ }^{1}$ Albeit disorderless, owing to intershell coupling, the electronic wavepackets propagate in an aperiodic system and complex multiple quantum interferences develop. It has been shown that the energy averaged propagation departs from ballistic conduction even in impurity-free incommensurate MWNT's (Refs. 12 and 13). In Ref. 14, the incommensurability was shown to break the selection rule of momentum conservation, thus enhancing intershell hopping. However, qualitative arguments conclude to minor effects in the conductance.

That issue is addressed here by considering triple-wall nanotubes with lengths of about $5 \mu \mathrm{m}$ and realistic tightbinding parameters. This allows us to draw quantitative conclusions within the experimental scope, by taking an interelectrode distance $L$ within the range $100 \mathrm{~nm}-1 \mu \mathrm{m}$.

In Fig. 4, intrinsic transport features of $\mathcal{D}(E, t)$ and $\mathcal{G}(E, L)$, for both commensurate and incommensurate triple wall nanotubes are presented. Several commensurate or incommensurate nanotubes with similar diameters but different helicities have been considered, giving similar results for electronic propagation. Hence only two representative cases are reported here. First the triple-wall $(15,15)$ @ $(10,10) @(5,5)$ with $C_{5 h}$ symmetry and composed of metallic and commensurate shells, is examined. As illustrated in Fig. 4 (left), over the whole spectrum, conduction remains ballistic and conductance (not shown here) results from the sum of all quantum channels available at a given 
energy (in particular, at $\mathrm{CNP} \mathcal{G}=6 \mathcal{G}_{0}$ ). Note that stepwise reduction of conductance has been predicted in absence of $C_{5 h}$ symmetry. $^{27}$

Next we focus on the triple wall $(15,15) @(17,0) @(6,4)$ made from an outer metallic shell and two semiconducting inner ones, with incommensurability between first neighbors. Differently to the previous case, the conduction modes are now strongly energy dependent. Indeed, at CNP, the massless subbands of the $(15,15)$ are weakly perturbed by the aperiodic potential due to the $(17,0)$, essentially because at such energy, there is no hybridization between states of different layers. Conduction is ballistic and the conductance is $\mathcal{G}=2 \mathcal{G}_{0}$. Conversely, as illustrated at $E=0.5 \gamma_{0}$ (Fig. 4, right), the wave packets spread over the several shells and a nonballistic regime, with $\mathcal{D} \sim t^{2 \eta-1}$ ( $\left.\eta \simeq 0.92\right)$, develops. At this energy, and more generally as soon as massive subbands are involved in conduction, the conductance evaluated from Eq. (3) downscales with inter-electrode distance and follows a general power law $\mathcal{G}(L) \sim e^{2} / h\left(L / L_{0}\right)^{(\eta-1) / \eta}\left(L_{0}\right.$ is constant at a given energy). One notes that the value of the exponent $\eta$ depends on both the Fermi level energy and the helical symmetries of the MWNT. It might happen that the value of $\eta$ approaches $1 / 2$, such that an effective "diffusivelike" regime would develop, having implications on Aharonov-Bohm phenomenon. ${ }^{9,12}$

Recent experiments ${ }^{11}$ have reported on anomalous scaling of conductance in boron-doped MWNT's. In the light of the present study, an important issue would be to investigate the gate-dependent length scaling of conductance, since a gate voltage will induce some shifting of the position of the Fermi level. ${ }^{22}$ Other experiments made on MWNT's at low bias found a nearly length independent conductance in the order of $\mathcal{G} \sim \mathcal{G}_{0}$ or $2 \mathcal{G}_{0},{ }^{6}$ suggesting that the Fermi level is located close to the CNP. However, by increasing the bias voltage, ${ }^{7}$ the measured conductance was found much lower than theoretically predicted in the ballistic regime. Our study points out that both the presence of defects or the multishell conduction in incommensurate systems can contribute to a strong reduction of conductance without contradiction with having a ballistic motion at lower energy.

In conclusion, we have reported on Fermi-leveldependent transport fingerprints in carbon nanotubes accounting for the possible role of defects or incommensurability. Our results demonstrate that unique gate-voltage dependent patterns are expected in the conductance of such fascinating quasi-one-dimensional molecular systems.

This work was supported by the Micro and Nanotechnology Program from French Ministry of Research under the grant RTB: Post CMOS moléculaire $200 \mathrm{~mm}$. A.R. thanks support from the EC research training network COMELCAN (Grant No. HPRN-CT-2000-00128), Spanish MCyT (Grant No. MAT2001-0946), M-DNA (Grant No. IST-2001-38051), and Universidad del País Vasco (Grant No. 9/UPV 00206.215-13639/2001).
*Author to whom correspondence should be addressed: Email address: ftriozon@cea.fr

†Email address: sroche@cea.fr

${ }^{1}$ R. Saito et al., Physical Properties of Carbon Nanotubes (Imperial College, London, 1998).

${ }^{2}$ T. Ando et al., J. Phys. Soc. Jpn. 67, 2857 (1998).

${ }^{3}$ P.L. McEuen et al., Phys. Rev. Lett. 83, 5098 (1999).

${ }^{4}$ C.T. White and T.N. Todorov, Nature (London) 393, 240 (1998).

${ }^{5}$ A. Bachtold et al., Phys. Rev. Lett. 84, 6082 (2000).

${ }^{6}$ S. Frank et al., Science 280, 1744 (1998); A. Urbina et al., Phys. Rev. Lett. 90, 106603 (2003).

${ }^{7} \mathrm{Ph}$. Poncharal et al., J. Phys. Chem. 106, 12104 (2002).

${ }^{8}$ P.G. Collins and Ph. Avouris, Appl. Phys. A: Mater. Sci. Process. 74, 329 (2002).

${ }^{9}$ A. Bachtold et al., Nature (London) 397, 673 (1999).

${ }^{10}$ K. Liu et al., Phys. Rev. B 63, 161404(R) (2001).

${ }^{11}$ V. Krstic et al., Phys. Rev. B 67, 041401(R) (2003).

${ }^{12}$ S. Roche et al., Phys. Rev. B 64, 121401(R) (2001); S. Uryu, ibid. 69, 075402 (2004).

${ }^{13}$ S. Roche et al., Phys. Lett. A 285, 94 (2001).

${ }^{14}$ Y.-G. Yoon et al., Phys. Rev. B 66, 073407 (2002).

${ }^{15}$ K.-H. Ahn et al., Phys. Rev. Lett. 90, 026601 (2003).

${ }^{16}$ M. Kociak et al., Phys. Rev. Lett. 89, 155501 (2002).

${ }^{17}$ S. Rosenblatt et al., Nano Lett. 2, 869 (2002); H.J. Dai et al., J.
Phys. Chem. B 103, 11246 (1999); J. Appenzeller et al., Phys. Rev. Lett. 89, 126801 (2002).

${ }^{18}$ R. Saito et al., J. Appl. Phys. 73, 494 (1993); J.C. Charlier and J.P. Michenaud, Phys. Rev. Lett. 70, 1858 (1993); Ph. Lambin et al., Comput. Mater. Sci. 2, 350 (1994); Y.K. Kwon and D. Tománek, Phys. Rev. B 58, R16001 (1998).

${ }^{19}$ A. Hansson et al., Phys. Rev. B 62, 7639 (2000).

${ }^{20}$ H.J. Choi and J. Ihm, Phys. Rev. Lett. 84, 2917 (2000); A. Rochefort and Ph. Avouris, J. Phys. Chem. A 104, 9807 (2000); P.E. Lammert et al., Phys. Rev. Lett. 87, 136402 (2001); J.J. Palacios et al., ibid. 90, 106801 (2003).

${ }^{21}$ S. Roche and D. Mayou, Phys. Rev. Lett. 79, 2518 (1997); S. Roche and R. Saito, ibid. 87, 246803 (2001).

${ }^{22}$ M. Krüger et al., Appl. Phys. Lett. 78, 1291 (2001).

${ }^{23}$ F. Triozon et al., Phys. Rev. B 65, 220202(R) (2002).

${ }^{24}$ C. Leforestier et al., J. Comput. Phys. 94, 59 (1991); F. Triozon et al., RIKEN Rev. 29, 73 (2000).

${ }^{25}$ T. Hertel and G. Moos, Phys. Rev. Lett. 84, 5002 (2000).

${ }^{26} \mathrm{R}$. Egger et al., in Interacting Electrons in Nanostructures, edited by R. Haug and H. Schoeller, Lecture Notes in Physics, Vol. 579 (Springer-Verlag, Berlin, 2001), p. 125.

${ }^{27} \mathrm{Ph}$. Lambin et al., Phys. Rev. B 62, 5129 (2000); S. Sanvito et al., Phys. Rev. Lett. 84, 1974 (2000). 